\title{
Eficiência e Produtividade dos Gastos, Ações e Serviços de Saúde nos Municípios Mais Populosos do Ceará no Contexto da EC 95/2016
}

Valério de Oliveira, Antonio Rafael; Cavalcante Mota, Samuel; Carvalho de Vasconcelos, Alessandra Eficiência e Produtividade dos Gastos, Ações e Serviços de Saúde nos Municípios Mais Populosos do Ceará no Contexto da EC 95/2016

Administração Pública e Gestão Social, vol. 14, núm. 1, 2022

Universidade Federal de Viçosa, Brasil

Disponible en: https://www.redalyc.org/articulo.oa?id=351569604010

\section{(ㄷ) (1) $(9 \Theta$}

Esta obra está bajo una Licencia Creative Commons Atribución-NoComercial-SinDerivar 4.0 Internacional, 


\title{
Eficiência e Produtividade dos Gastos, Ações e Serviços de Saúde nos Municípios Mais Populosos do Ceará no Contexto da EC 95/2016
}

\author{
Efficency and Productivity of Health Care Spending, Actions and Services in Major Municipalities in Ceará under \\ Constitutional Amendment 95/2016 \\ La Eficiencia y la Productividad de los Gastos, Acciones y Servicios de Salud en los Municipios más Poblados de \\ Ceará en el Contexto de la EC 95/2016
}

\author{
Antonio Rafael Valério de Oliveira \\ Universidade Federal do Ceará, Brasil \\ rafaelvalerio65@gmail.com \\ Samuel Cavalcante Mota \\ Universidade Federal do Ceará, Brasil \\ samuelcmota@hotmail.com \\ Alessandra Carvalho de Vasconcelos \\ Universidade Federal do Ceará, Brasil \\ alevasconcelos.ufc@gmail.com
}

Redalyc: https://www.redalyc.org/articulo.oa? $\mathrm{id}=351569604010$

Recepción: 21 Junio 2021

Aprobación: 20 Agosto 2021

Publicación: 08 Enero 2022

\section{Resumo:}

Objetivo da pesquisa: Analisar a eficiência e a produtividade dos gastos, ações e serviços de saúde nos municípios mais populosos do Ceará antes e depois da EC 95/2016.

Enquadramento teórico: $\mathrm{O}$ estudo aborda a destinação eficiente dos recursos da saúde, que tem se transformado em um desafio para os gestores municipais preocupados em alcançar a universalidade da assistência à população, sobretudo, no contexto de crise econômica e de austeridade fiscal implementado pela EC 95/2016.

Metodologia: O estudo utiliza a Análise Envoltória de Dados (DEA) e o Índice de Produtividade Malmquist (IPM), usando o modelo BCC, orientados para output, para aferir eficiência e produtividade dos 36 municípios mais populosos do Ceará, com dados de 2015 a 2018.

Resultados: Depois da vigência da EC 95/2016 há maior quantidade de municípios eficientes e com maiores escores de eficiência, não sendo identificado correlação entre a eficiência e os indicadores PIB per capita e IEGM_Saúde. Ademais, houve aumento na produtividade total dos municípios nos anos depois da EC.

Originalidade: A pesquisa avalia a eficiência e a produtividade dos gastos com saúde nos municípios cearenses e correlaciona a eficiência com os indicadores PIB per capita e IEGM_Saúde, esse último, ainda não explorado por estudos anteriores. O artigo também contribui para subsidiar a elaboração das políticas públicas de saúde no Ceará, ao explorar a eficiência e a produtividade dos municípios no contexto da EC 95/2016.

Contribuições teóricas e práticas: Em termos teóricos, assinala-se que a EC 95/2016 afetou positivamente a eficiência e a produtividade dos gastos da saúde, entretanto, há indicação de ocorrência de impactos negativos ao longo de sua vigência. $\mathrm{Na}$ prática, sinaliza-se que os municípios com melhor situação econômica, absorvida pelo PIB per capita, não são os mais eficientes na aplicação dos recursos em saúde, bem como os melhores IEGM_Saúde também não são.

Palavras-chave: Eficiência, Produtividade, Gastos públicos em saúde, Municípios cearenses.

\section{Aвstract:}

Research objective: To evaluate the efficiency and productivity of health care spending, actions and services in the most populous municipalities of the State of Ceará (Brazil), under constitutional amendment 95/2016 (EC95).

Teoretical framework: The study addresses the allocation of health care resources, a challenge for municipal managers concerned with the universality of public health care during times of economic downturn and fiscal austerity imposed by EC95. 
Methodology: Output-oriented data envelopment analysis, the Malmquist Index, and BCC models were used to measure the efficiency and productivity of health care spending, actions and services in the 36 most populous municipalities of Ceará, covering the period 2015-2018.

Results: After the passage of EC95, more municipalities were classified as efficient, and the overall efficiency score was higher, but no correlation was found between efficiency and per capita GDP or 'IEGM_Saúde' (a municipal management index). Total productivity also increased in the years after EC95.

Originality: Earlier studies have analyzed the association between per capita GDP and the efficiency of health care spending in Ceará, but this is the first study to evaluate 'IEGM_Saúde' in this context. Moreover, our analysis of the influence of EC95 on efficiency and productivity offers novel subsidies for public health policy making in Ceará.

Teoretical and practical contributions: In theory, EC95 favors the efficiency and productivity of health care spending, although some negative impacts have been reported. Municipalities with higher per capita GDP and 'IEGM_Saúde' are not necessarily more efficient at allocating health care resources.

KEYWORDs: Efficiency, Productivity, Public health care spending, Municipalities of Ceará.

\section{RESUMEN:}

Objetivo de la investigación: Analizar la eficiencia y la productividad de los gastos, acciones y servicios de salud en los municipios más poblados de Ceará antes y después de la EC 95/2016.

Aportación teórica: El estudio aborda la asignación eficiente de los recursos de la salud, que se ha convertido en un desafío para los gestores municipales preocupados por lograr la universalidad de la atención a la población, especialmente en el contexto de crisis económica y de austeridad fiscal implementado por la EC 95/2016.

Metodología: El estudio utiliza el Análisis por Envoltura de Datos (DEA) y el Índice de Productividad de Malmquist (IPM), utilizando el modelo BCC, orientados para output, para medir la eficiencia y la productividad de los 36 municipios más poblados de Ceará con datos de 2015 a 2018.

Resultados: Luego de la vigencia de la EC 95/2016 hay una mayor cantidad de municipios eficientes y con mayores puntajes de eficiencia, sin que se identifique correlación entre la eficiencia y los indicadores PIB per cápita e 'IEGM_Saúde'. Además, hubo un aumento en la productividad total de los municipios en los años posteriores de la EC.

Originalidad: La investigación evalúa la eficiencia y la productividad de los gastos con salud en los municipios cearenses y correlaciona la eficiencia con los indicadores PIB per cápita e 'IEGM_Saúde'. Este último todavía no ha sido explorado por estudios anteriores. El artículo también contribuye para apoyar el diseño de políticas públicas de salud en Ceará al explorar la eficiencia y la productividad de los municipios en el contexto de la EC 95/2016.

Contribuciones teóricas y prácticas: En términos teóricos, se observa que la EC 95/2016 afectó positivamente la eficiencia y la productividad de los gastos de la salud, sin embargo, hay indicios de la ocurrencia de impactos negativos a lo largo de su vigencia. En la práctica se indica que los municipios con mejor situación económica, absorbida por el PIB per cápita, no son los más eficientes en la aplicación de los recursos en salud, así como los mejores 'IEGM_Saúde' tampoco lo son.

Palabras clave: Eficiencia, Productividad, Gastos públicos en salud, Municipios cearenses.

\section{INTRODUÇÃO}

O setor de saúde requer zelo na formulação das políticas públicas, pois, tem como principal missão cuidar da vida humana. Assim, o grande desafio consiste em associar os resultados, considerando-se os limitados recursos disponíveis. No Brasil, as políticas públicas de saúde possuem como um dos pilares primordiais a descentralização das ações e dos recursos até o nível municipal (Marinho, 2003).

Vieira e Benevides (2016) mencionam que desde a criação do Sistema Único de Saúde (SUS), o compromisso de consolidação do programa configurou-se repetidamente nos diferentes governos; entretanto, muitos são os desafios para a garantia de seu financiamento. Nesse contexto, a alocação eficiente dos recursos no campo da saúde tem se transformado em um desafio para os gestores públicos, para o alcance da universalidade e da integralidade da assistência à saúde dispostas na legislação (Mazon, Mascarenhas, \& Dallabrida, 2015).

Devido à escassez dos recursos, os gastos devem ser feitos de modo eficiente, visando a atender às demandas sociais (Scarpin, Macedo, Starosky, \& Rodrigues, 2012). Ademais, em virtude da obrigação de cumprir a descentralização, o governo federal precisou criar mecanismos regulatórios de transferência de 
responsabilidades e de recursos para os governos municipais, para a gestão da saúde no âmbito local (Mattos, Rocha, Novaes, Arvate, \& Orellano, 2009). Outro mecanismo regulatório foi introduzido pela Emenda Constitucional n. 29, que fixou porcentagens mínimas de aplicação em ações e serviços de saúde pelos entes federados, em relação às receitas próprias. No caso dos municípios, foi estabelecido o mínimo de $15 \%$, com alcance progressivo até o ano 2004 (Emenda Constitucional n. 29, 2000). Sobretudo devido ao advento do Novo Regime Fiscal (NRF), instituído pela Emenda Constitucional n. 95 (Emenda Constitucional n. 95, 2016), Vieira e Benevides (2016) advertem que o roteiro de baixa priorização da saúde nos orçamentos e no processo de execução terá piora com o congelamento do gasto primário federal por 20 anos, podendo gerar profundos impactos no financiamento da saúde.

O NRF retirou, na prática, o governo federal do pacto de financiamento tripartite do SUS e enfraqueceu a sua função na coordenação nacional (Costa \& Lago, 2020). Os governos municipais enfrentam desafios políticos e econômicos que demandarão melhorias nos serviços locais, some-se a isso os impactos da austeridade fiscal que irá, substancialmente, reduzir os gastos com programas de assistência social nos próximos 20 anos (Rasella et al., 2018).

No caso dos municípios cearenses, Machado, Irffi e Benegas (2011) apontam uma baixa eficiência técnica no tocante à aplicação dos gastos públicos com saúde. Nunes e Souza (2019) afirmam inclusive que o Ceará tem enfrentado diversos problemas relacionados à infraestrutura no segmento da saúde, conquanto contribua diretamente para outras áreas, como educação e desenvolvimento econômico e social.

Pesquisas recentes dedicadas à análise do desempenho de municípios (Teles, 2018; Nunes \& Sousa, 2019; Macêdo, Reis, Silveira, \& Salla, 2019) têm demonstrado que, além da grande variação dos níveis de prestação de serviços na esfera municipal, em sua grande maioria apresentaram baixa eficiência e produtividade na implementação de políticas públicas.

Considerando-se que a Emenda Constitucional n. 95 foi aprovada recentemente, e que seus impactos sobre o nível de eficiência e de produtividade municipal ainda não foram avaliados, emerge a seguinte questãoproblema: Qual o nível de eficiência e de produtividade dos gastos, ações e serviços de saúde dos municípios mais populosos do Ceará no contexto da EC 95/2016? Destarte, constitui o objetivo principal deste estudo analisar a eficiência e a produtividade dos gastos, ações e serviços de saúde nos municípios mais populosos do Ceará antes e depois da EC 95/2016. Adicionalmente, buscou-se examinar a correlação entre o nível de eficiência dos municípios e os indicadores de desenvolvimento social PIB per capita e IEGM_Saúde.

Estudos que avaliam a eficiência da gestão pública nas áreas de saúde, educação e assistência social frequentemente utilizam indicadores de desenvolvimento social para mensurar o bem-estar social. A maioria deles adota o Produto Interno Bruto (PIB), embora isoladamente o indicador possa ser insuficiente para se verificar o grau de bem-estar da sociedade (Silva, Alves, Luca, \& Vasconcelos, 2019). Outros indicadores têm a característica de mensurar o desenvolvimento socioeconômico municipal, como o Índice de Desenvolvimento Humano (IDH) e o Índice Firjan de Desenvolvimento Municipal (IFDM) (Leite \& Fialho, 2015). Nessa perspectiva, o presente estudo utiliza o Índice de Efetividade da Gestão Municipal Dimensão Saúde (IEGM_Saúde), do Tribunal de Contas do Estado do Ceará, possui sete dimensões, e é apurado anualmente (Tribunal de Contas do Estado do Ceará [TCE-CE], 2019).

Destaca-se que o IEGM_Saúde é obtido por meio de aplicação de questionário junto aos municípios e visa medir a efetividade das gestões municipais, sendo que os dados obtidos são, posteriormente, confirmados por técnicos do tribunal de contas estadual. O índice possibilita a comparação de desempenho, a identificação de boas práticas e, consequentemente, contribui para a melhoria da eficiência municipal (Araújo, Bezerra Filho, Teixeira, \& Reina, 2015). Dessa forma, seguindo o estudo recente desenvolvido por Ramos, Silva, Cerqueira e Queiroz (2021), esta pesquisa parte do pressuposto que o Índice de Efetividade de Gestão Municipal (IEGM) é um indicador de performance efetivo para essa avaliação.

A Análise Envoltória de Dados (DEA) foi utilizada para analisar o grau de eficiência dos municípios, sendo cada um deles considerado no modelo como uma Unidade Tomadora de Decisão(DMU), responsável 
por utilizar recursos (inputs) em prol da obtenção de produtos (outputs), combinado com o Índice de Produtividade de Malmquist (IPM). Na sequência, foram aplicados testes de correlação para verificar o relacionamento entre eficiência e os indicadores PIB per capita e IEGM_Saúde dos 36 municípios cearenses mais populosos.

A eficiência da administração pública tem sido objeto de várias pesquisas nacionais. Algumas delas, recentemente, deram ênfase à eficiência dos estados (Andrett, Lunkes, Rosa, \& Brizolla, 2018; Silva et al., 2019), enquanto outras, concentram-se na eficiência dos gastos com educação nos municípios (Rosano-Peña, Albuquerque, \& Márcio, 2012; Scarpin et al., 2012) e na eficiência dos gastos com saúde nos municípios (Alves \& Costa, 2013; Macêdo et al., 2019; Mazon et al., 2015; Nunes \& Sousa, 2019).

As pesquisas de Duarte e Diniz (2018), Macêdo et al. (2019) e Nunes e Sousa (2019), são as que guardam maior proximidade com o presente estudo. Contudo, esta pesquisa inova ao avaliar a eficiência e a produtividade da saúde nos municípios cearenses e correlacioná-la com os indicadores PIB per capita e IEGM_Saúde, este último, ainda não explorado nos estudos prévios. Ademais, também contribui para enriquecer a literatura e subsidiar a elaboração das políticas públicas de saúde no Ceará, ao explorar a temática desde o início da vigência da EC 95.

Segundo Vieira e Benevides (2016), a EC 95 apresenta obstáculos à concretização do direito à saúde, incentivando os que dispõem de recursos a contratar plano de saúde; aqueles que compreendem os seus direitos, as responsabilidades do Estado e os meios de exigi-los, a buscar a judicialização; e, enquanto isso, os mais vulneráveis estarão sujeitos à escassez da oferta e à baixa qualidade dos serviços públicos de saúde, crescentemente mais precarizados.

Por outro lado, com base no "mito da contração fiscal expansionista", Alesina, Bariero, Fávero, Giavazzi e Paradisi (2017) asseguram que o NRF estimulará os investimentos privados e a retomada das atividades econômicas por meio do ajuste das contas públicas. Assim, pretende-se preencher uma lacuna na literatura, quer seja pela observação dos impactos da legislação, mediante implementação da emenda, quer seja pela possível correlação entre a eficiência e o IEGM_Saúde.

\section{EFICIÊNCIA E PRODUTIVIDADE NA GESTÃO DA SAÚDE}

A partir da análise sobre produtividade de Debreu (1951), deu-se início aos estudos acerca da Teoria Econômica da Eficiência. Mediante aprofundamento do tema por Farrel (1957), surge a afirmação de que a eficiência da organização consiste em gerar, com um dado conjunto de insumos (inputs), o máximo possível em produtos (outputs).

Inicialmente, Farrel (1957) baseou-se no caso de uma organização que fabricava um produto com dois fatores de produção, cujo processo produtivo apresentava Retornos Constantes de Escala (RCE). As medições da eficiência se baseavam na premissa de que a empresa conhecia detalhadamente sua função de produção, situação que exigia uma perfeita definição dos processos envolvidos, condição que, em alguns casos, envolvia uma extrema complexidade (Farrel, 1957).

Diante dessa dificuldade, o autor estimou uma função de produção, considerando, nas informações observadas, os inputs. outputs para algumas organizações; na sua visão, o fundamento da aferição de eficiência técnica de uma empresa hipotética era a média ponderada de um número apropriado de empresas observadas (Farrel, 1957).

Com base na pesquisa de Charnes, Cooper e Rhodes (1978), direcionada para a avaliação das atividades de entidades sem fins lucrativos em programas públicos, foi possível generalizar os estudos de Farrel (1957) para a medida de eficiência com vários insumos e vários produtos.

A concepção de Charnes et al. (1978), distinguindo-se das pesquisas de Farrel (1957), não requer conhecimento prévio da função de produção da empresa, isto é, independe das unidades de medida e dos 
pesos das diversas variáveis. Assim, a DEA se torna imprescindível para aplicação do modelo de avaliação da eficiência e as unidades produtivas devem ser semelhantes em relação à utilização de insumos e produtos.

Segundo Silva et al. (2019), a DEA calcula a eficiência das DMUs na razão entre os produtos e os insumos, fazendo uso da programação matemática linear para estimar a fronteira da capacidade de produção. $\mathrm{O}$ resultado obtido por meio do modelo varia entre 0 e 1 , em que " 0 " indica que a DMU é ineficiente, e " 1 " significa que a DMU é eficiente.

O método possibilita comparar os insumos (inputs) e os produtos (outputs) de cada unidade, determinando os respectivos índices de eficiência relativa. Nesse sentido, pode-se identificar recursos ociosos ou inutilizados, bem como auxiliar na formação de políticas de redução de custos nas instituições (Santos, Freitas, \& Flach, 2015).

Para analisar o efeito da EC 95/2016, faz-se necessário considerar a dinâmica da eficiência ao longo do tempo e a principal metodologia para isso é o Índice de Produtividade de Malmquist (IPM), visto que não se utiliza dos preços para agrupar as distintas entradas e saídas (Bogetoft \& Otto, 2011). A DEA e o IPM buscam estimar os níveis de eficiência relativa e de eficiência dinâmica (Rosano-Peña, Albuquerque, \& Daher, 2012).

Aferir a eficiência no setor de saúde é complexo, pois, segundo Lopes, Toyoshima e Gomes (2009), há alguns problemas básicos no SUS, como o financiamento do setor, que tem sido insuficiente para cobrir os propósitos de universalização, integralidade e equidade. Além de se gastar pouco com saúde, muitas vezes esses recursos não vêm sendo alocados da forma mais eficiente possível.

O processo de avaliação de uma política pública constitui um desafio para o gestor, haja vista que existe uma quantidade de variáveis, muitas vezes veladas, que acabam impactando o pleno diagnóstico dos problemas e das potencialidades do que vem sendo estudado. $\mathrm{Na}$ área da saúde não poderia ser diferente, haja vista a complexidade da área, de modo que analisar o SUS torna-se uma tarefa complexa, pois há variáveis qualitativas que precisam ser consideradas na análise da eficiência do sistema (Costa \& Rodrigues, 2016).

Em se tratando especificamente do Ceará, Silva (2010) assevera que o setor de saúde enfrentou crises em anos recentes, a saber: a carência de leitos de Unidade de Tratamento Intensivo (UTI), a demora demasiada nos atendimentos, a falta de médicos e as longas filas de pacientes esperando a realização de procedimentos cirúrgicos as principais dificuldades.

\section{ESTUDOS CORRELATOS}

A avaliação da eficiência e da produtividade tem sido realizada em pesquisas estrangeiras e nacionais relacionadas aos gastos públicos com saúde. Esse fato pode ser explicado por Kohl, Schoenfelder, Fügener e Brunner (2019), pois, ao revisarem publicações que utilizaram a DEA, catalogaram 262 artigos que fizeram uso do método no setor de saúde entre 2005 e 2016, sinalizando que há, nos últimos anos, um número crescente de publicações. Internacionalmente, de modo exemplificativo, citam-se os estudos de Asandului, Roman e Fatulescu (2014), Lobo e Araújo (2017) e Sun, Ahn, Lievens e Zeng (2017), sobre a eficiência da administração pública com os gastos em saúde.

Entre os estudiosos brasileiros, Nunes e Sousa (2019) mensuraram o nível de eficiência técnica e de escala do IFDM na área da saúde para 162 municípios cearenses e agregaram os níveis de eficiência por mesorregião, PIB per capita e população. Utilizando dados de 2013, aplicaram a DEA e constataram que uma parcela majoritária dos municípios apresentou problemas quanto à escala de produção, sendo resultante da presença de retornos decrescentes à escala. Ademais, os resultados indicam que os municípios de regiões mais desenvolvidas, com PIB per capita mais elevado e mais populosos, não necessariamente gerenciam seus recursos da forma mais eficiente.

Macêdo et al. (2019) analisaram a relação entre transparência fiscal e eficiência dos gastos com educação e saúde nos 184 municípios cearenses, utilizando medidas de estatística descritiva, análise de correlação e a DEA. Por meio dos inputs receitas correntes e receitas tributárias e dos outputs atenção básica, assistência 
hospitalar e ambulatorial e vigilância sanitária, constataram que Ararendá, Crateús e Fortaleza se mostraram mais eficientes. No extremo oposto, Quiterianópolis, Senador Sá e Tururu foram os menos eficientes.

Barbosa e Sousa (2015) identificaram os escores de eficiência técnica e de escala do Índice de Desempenho do Sistema Único de Saúde (IDSUS) e agruparam 1.790 municípios do Nordeste em seis clusters com semelhantes características socioeconômicas, condições sociais e estrutura do sistema de saúde. Os resultados apontam que os municípios com melhores índices - referentes às características socioeconômicas (IDSE), às condições sociais (ICS) e à estrutura do sistema de saúde do município (IESSM) -, são os que, em média, apresentam os maiores escores de eficiência técnica e de escala do IDSUS.

Lobo e Araújo (2017) examinaram as relações entre os níveis de atenção à saúde nas capitais brasileiras, com ênfase nos cuidados primários, secundários e terciários, utilizando dados de 2008 a 2013. Constataram que oito, das 27 capitais (incluindo Brasília), são efetivamente eficientes. Os níveis primário e secundário aumentaram as respectivas pontuações médias no período, mas apenas o terciário obteve uma mudança tecnológica positiva.

Queiroz, Silva, Figueiredo e Vale (2013b) analisaram a aplicação de recursos direcionados à saúde, no Rio Grande do Norte. Constataram que os municípios contemplados com os menores aportes de recursos, de maneira geral, revelaram-se menos eficientes, sendo que apenas $26 \%$ foram eficientes. Concluíram que a obtenção da eficiência nos indicadores relacionados à saúde pública municipal, subordina-se vigorosamente à dotação de recursos.

Quanto à produtividade, Zhang, Wang, Jiang e Wang (2018) analisaram as alterações de produtividade e eficiência na alocação de recursos da atenção primária à saúde da China, explorando formas de melhorar a situação atual. Para medir a eficiência e a produtividade, os autores utilizaram a DEA e o IPM.

Duarte e Diniz (2018) averiguaram em que medida o volume de gastos públicos em saúde afeta o aumento da eficiência e da produtividade dos serviços de média e alta complexidade nos estados brasileiros, combinando DEA e IPM. Os resultados indicam que os estados com maiores gastos, em média, não puderam ser considerados os mais eficientes, indicando que pode haver relação negativa entre crescimento dos gastos e a eficiência.

Também foi foco de atenção de alguns estudos os ajustes fiscais e as reformas implementados recentemente. Entre as pesquisas que deram ênfase à saúde, destacam-se as de Rasella et al. (2018) e Vieira e Benevides (2016).

A literatura aponta que poucos municípios vêm utilizando os recursos de maneira racional, necessitando de aprimoramento na relação custo/benefício/retorno dos recursos públicos destinados à população (Macedo et al., 2019; Machado et al., 2011; Nunes \& Sousa, 2019; Silva, 2010), sobretudo após o início de vigência da EC 95, em virtude das divergências quanto aos seus efeitos.

\section{METODOLOGIA}

A população da pesquisa reúne os 36 municípios do Ceará com mais de 50 mil habitantes, segundo estimativa da Resolução IBGE n. 3, de 26 de agosto de 2019, com data de referência em 01/07/2019, para os fins previstos no inciso VI do Art. $1^{\circ}$ da Lei n. 8.443, de 16/07/1992 (Instituto Brasileiro de Geografia e Estatística [IBGE], 2019). O estudo dos municípios mais populosos segue procedimento semelhante ao adotado por Galoni e Camargo (2016), que analisaram a eficiência em termos de educação com o Ensino Fundamental.

Para Peña (2008), a eficiência é alcançada por meio de uma combinação ótima entre os insumos (inputs), gerando o máximo de produtos (outputs). A DEA é um modelo não paramétrico aplicado com sucesso nos estudos da eficiência na administração pública - utilizado para comparar vários tipos de entidade, como escolas, hospitais e universidades - e adotado para analisar o desempenho de DMUs que utilizam os mesmos tipos de insumo para produzir os mesmos bens e/ou serviços (Peña, 2008). 
O modelo Retornos Variáveis à Escala, proposto por Banker, Charnes e Cooper (1984) e nomeado BCC (DEA-BCC) foi utilizado no estudo, visto que se pretende, a partir dos mesmos níveis de insumo, a obtenção dos melhores resultados. A opção pelo modelo se justifica por não haver proporcionalidade entre os inputs . outputs nos municípios estudados, já que apresentar maiores valores aplicados não garantirá, por si só, maior eficiência na gestão dos recursos públicos da saúde.

A aplicação do modelo trata de obter resultados que visem à maximização do desempenho dos municípios cearenses mais populosos, mantendo-se estáveis os níveis de indicadores de insumos no setor de saúde, conforme empregado por Andrade, Serrano, Bastos e Franco (2017), Fonseca e Ferreira (2009) e Queiroz et al. (2013b).

A população do estudo se enquadra no conceito de DMU, já que apresenta semelhanças tanto nos insumos utilizados como nos serviços prestados. Isso pode ser comprovado pela utilização do método DEA em vários estudos que tiveram como objeto municípios ou estados, parte deles evidenciada no referencial teórico.

A Tabela 1 apresenta a relação de DMUs utilizadas neste estudo, com a identificação dos municípios e respectivas populações, conforme as estimativas do IBGE (2019).

Tabela 1: Ranking dos 36 municípios mais populosos do Ceará

\begin{tabular}{ccc|ccc}
\hline $\mathbf{N}^{0}$ de Ordem & Município & População & $\mathbf{N}^{\circ}$ de Ordem & Município & População \\
\hline 1 & Fortaleza & 2.669 .342 & 19 & Pacajus & 72.203 \\
2 & Caucaia & 361.400 & 20 & Cascavel & 71.743 \\
3 & Juazeiro do Norte & 274.207 & 21 & Icó & 68.018 \\
4 & Maracanaú & 227.886 & 22 & Horizonte & 67.337 \\
5 & Sobral & 208.935 & 23 & Camocim & 63.661 \\
6 & Crato & 132.123 & 24 & Acaraú & 62.641 \\
7 & Itapipoca & 129.358 & 25 & Morada Nova & 61.890 \\
8 & Maranguape & 128.978 & 26 & Viçosa do Ceará & 60.889 \\
9 & Iguatu & 102.498 & 27 & Barbalha & 59.732 \\
10 & Quixadá & 877.28 & 28 & Limoeiro do Norte & 59.540 \\
11 & Pacatuba & 83.432 & 29 & Tauá & 58.859 \\
12 & Quixeramobim & 81.082 & 30 & Trairi & 55.918 \\
13 & Aquiraz & 80.271 & 31 & Granja & 54.748 \\
14 & Russas & 78.194 & 32 & Boa Viagem & 54.470 \\
15 & Canindé & 76.997 & 33 & Acopiara & 54.270 \\
16 & Tianguá & 75.946 & 34 & Eusébio & 53.618 \\
17 & Crateús & 75.074 & 35 & Beberibe & 53.573 \\
18 & Aracati & 74.547 & 36 & Itapajé & 52.675 \\
\cline { 3 - 5 } & & Total & & $\mathbf{6 . 0 3 3 . 7 8 3}$ \\
\hline
\end{tabular}

Fonte: Adaptado do IBGE (2019).

A coleta de dados referentes aos inputs outputs alusivos ao período de 2015 a 2018 (dois anos antes e dois depois do início da vigência da EC 95), bem como dos indicadores PIB per capita e IEGM_Saúde de 2018 dos municípios, foi realizada no período de agosto a outubro de 2020. O Quadro 1 detalha as variáveis utilizadas no modelo DEA. 
Quadro 1: Variáveis utilizadas no modelo DEA

\begin{tabular}{|c|c|c|c|}
\hline Inputs & Descrição & Fonte & Referência \\
\hline GasPubPC & $\begin{array}{c}\text { Gasto público per capita } \\
\text { com saúde }\end{array}$ & IBGE e MS & $\begin{array}{c}\text { Machado et al. (2011); Mont Mor e Barbosa } \\
\text { (2018); Teles (2018); Nunes e Souza (2019) }\end{array}$ \\
\hline PercASPS & $\begin{array}{c}\text { Percentual de aplicação } \\
\text { em Ações e Serviços } \\
\text { Público de Saúde (ASPS) }\end{array}$ & MS & $\begin{array}{c}\text { Lei Complementar n. 141, de 13 de janeiro de } \\
\text { 2012; Teles (2018) }\end{array}$ \\
\hline EquiSau/1000 & $\begin{array}{c}\text { Equipes de saúde } / 1000 \\
\text { habitantes }\end{array}$ & DATASUS & $\begin{array}{c}\text { Mazon, Mascarenhas e Dallabrida (2015); } \\
\text { Flach, Matos e Mendes (2017) }\end{array}$ \\
\hline Outputs & Descrição & Fonte & Referência \\
\hline Cobvac & Cobertura vacinal & DATASUS & Andrett et al. (2018) \\
\hline Imort & $\begin{array}{c}\text { Inverso da taxa de } \\
\text { mortalidade infantil }\end{array}$ & IBGE / SESA / IPCE & $\begin{array}{c}\text { Silva, Moretti e Schuster (2016); } \\
\text { Mazon, Mascarenhas e Dallabrida (2015); } \\
\text { Teles (2018) }\end{array}$ \\
\hline Leitos/1000 & $\begin{array}{c}\text { Número de leito } \\
\text { SUS/1000 }\end{array}$ & DATASUS & Queiroz et al. (2013a) \\
\hline
\end{tabular}

A escolha dos inputs se deu pelo fato de a Constituição Federal (Constituição da República Federativa do Brasil, 1988) e suas alterações, estipular gastos mínimos com a saúde, além de destacar que esse segmento é fundamental para a dignidade humana (Silva et al., 2019), sendo também considerada por Andrett et al. (2018), Mont Mor e Barbosa (2018) e Silva et al. (2019). Na escolha dos outputs, foram consideradas as variáveis que levam em conta a saúde preventiva e a curativa, pois, consoante Fonseca e Ferreira (2009), constituem referências fundamentais para a mensuração da eficiência na área da saúde.

Foi aplicado o Índice de Produtividade Malmquist (IPM). Begotoff e Otto (2011) advogam que o principal método para averiguar a dinâmica da eficiência no tempo é o Índice IPM (Malmquist Index), visto que não utiliza preços para aglutinar as entradas e as saídas. Os modelos de eficiência são classificados em categorias. Uma delas é a variação nomeada de dados, na qual existem dados baseados em probabilidade e em séries temporais. A segunda categoria é nomeada de alteração da eficiência ao longo do tempo (Rosano-Peña, Albuquerque \& Daher, 2012; Sánchez, 2018).

O IPM oportuniza comparar a eficiência em períodos distintos, sendo decomposto em dois componentes: a variação de eficiência técnica (catch-up) e a variação tecnológica (frontier-shift effect). Assim, possibilita verificar se o deslocamento da fronteira de eficiência é decorrente do aumento da eficiência técnica ou da incorporação de novas tecnologias (Marinho \& Ataliba, 2000).

Adicionalmente, e visando à análise de correlação entre os níveis de eficiência dos municípios e os respectivos indicadores de desenvolvimento social na vigência da EC 95, consideraram-se o PIB per capita e o IEGM_Saúde do TCE-CE, ambos referentes a 2018. Os dados utilizados na pesquisa foram extraídos do Siops, do Datasus, do IBGE e do TCE.

Para o alcance dos objetivos propostos, foram realizados testes de normalidade das variáveis e testes de correlação de Pearson com correção bootstrap, já que os dados não são ordinais. Para a análise dos dados por meio do método DEA e do IPM, foi utilizado o software Frontier Analyst, enquanto para a realização dos testes de normalidade e correlação, utilizou-se o IBM SPSS Statistics 20. 


\section{RESULTADOS E DISCUSSÃO}

\subsection{Análise descritiva}

A Tabela 2 apresenta a análise descritiva das variáveis de inputs e outputs dos 36 municípios cearenses mais populosos utilizadas no modelo DEA no quadriênio estudado (2015 a 2018), sendo dois anos antes e dois depois da vigência da EC 95/2016.

Tabela 2: Estatística descritiva das variáveis de inputs e outputs

\begin{tabular}{l|ccccc}
\hline \multirow{2}{*}{ Indicadores } & \multicolumn{5}{c}{ Antes da EC 95/2016 } \\
\cline { 2 - 6 } & Mínimo & Máximo & Média & Desvio-padrão & Coeficiente de variação (\%) \\
\hline GasPubPC (I) & 108.25 & 777,62 & 235,34 & 124,64 & 37,61 \\
PercASPS (I) & 14.38 & 35,00 & 24,00 & 5,07 & 18,74 \\
EquiSau/1000 (I) & 0,13 & 0,52 & 0,34 & 0,08 & 23,25 \\
Cobvac (O) & 33.35 & 157,18 & 82,62 & 31,02 & 30,25 \\
Imort (O) & 0,04 & 0,38 & 0,10 & 0,06 & 60,66 \\
NLeitos/1000 (O) & 0,24 & 5,78 & 1,49 & 1,11 & 57,71 \\
\hline \multirow{2}{*}{ Indicadores } & \multicolumn{5}{c}{ Depois da EC 95/2016 } \\
\cline { 2 - 6 } & Mínimo & Máximo & Média & Desvio-padrão & Coeficiente de variação (\%) \\
\hline GasPubPC (I) & 110,75 & 938,16 & 237,68 & 135,81 & 39,68 \\
PercASPS (I) & 15,12 & 34,97 & 24,00 & 5,09 & 21,43 \\
EquiSau/1000 (I) & 0,19 & 0,56 & 0,36 & 0,07 & 24,17 \\
Cobvac (O) & 53,06 & 94,78 & 75,31 & 8,52 & 28,02 \\
Imort (O) & 0,04 & 0,21 & 0,09 & 0,03 & 60,66 \\
NLeitos/1000 (O) & 0,23 & 5,84 & 1,47 & 1,21 & 56,72 \\
\hline
\end{tabular}

$\mathrm{Na}$ Tabela 2, verifica-se que nos 36 municípios os gastos com saúde apresentaram valor médio de $\mathrm{R} \$ 235,34$ antes e de $\mathrm{R} \$ 237,68$ depois da EC 95. Essa variável possibilita observar a disparidade entre o gasto per capita e o porte dos municípios, pois, enquanto Caucaia (361.400 hab.) gastou em média $R \$ 108$,25, Eusébio (53.618 hab.) dispendeu R $\$ 777,62$. Depois da EC, nota-se a mesma tendência, pois Itapipoca (129.358 hab.) gastou em média $R$ \$ 110,75 e Eusébio (53.618 hab.) empregou $\mathrm{R} \$ 938,16$ em média com saúde.

Referente à LC n. 141/2012, quase a totalidade dos municípios apresentou valor maior que o exigido, com exceção de Pacatuba, que em 2016 aplicou 14,38\%. Carvalho (2013) corrobora essa constatação ao assegurar que os estados e os municípios são os responsáveis finalísticos pelas ASPS, sendo que diante do subfinanciamento federal, os municípios aplicam acima do piso legal (15\%), alguns ultrapassando $20 \%$. Corroborando, Sousa, Pinhanez, Monte e Cavalcante (2020) demonstraram que a aplicação de recursos resultantes de impostos de acordo com a EC 29 é superior ao limite mínimo estabelecido de $15 \%$ nos municípios das quatro macrorregiões do estado de Pernambuco.

Apenas as variáveis "Gasto público per capita com saúde" e "Equipes de saúde / 1000 habitantes" obtiveram aumento depois da EC 95/2016, corroborando os prognósticos de Vieira e Benevides (2016) que estimaram que a EC provocará baixa priorização nos orçamentos e na execução da saúde, pois o congelamento do gasto primário federal por 20 anos ocasionará impactos no financiamento.

A “cobertura vacinal”, apresentou decréscimo de 82,62 para 75,31. A diminuição da imunização no Brasil vem ocorrendo em virtude da ausência de conhecimento acerca dos benefícios, da baixa escolaridade e da campanha de desinformação, conforme Militão e Mazieiro (2020). 


\subsection{Eficiência relativa dos municípios}

Para a distribuição das DMUs, considerando seu escore de eficiência, adaptaram-se as medidas de eficiência utilizadas por Barbosa e Souza (2015) e Nunes e Souza (2019). A Tabela 3 apresenta a distribuição das DMUs nos grupos formados e os respectivos limites.

Tabela 3: Grupos de DMUs por faixa de escores de eficiência (EE) antes e depois da EC 95/2016

\begin{tabular}{|c|c|c|c|c|}
\hline \multirow[b]{2}{*}{ Faixa } & \multicolumn{2}{|c|}{ Média antes da EC 95/2016 } & \multicolumn{2}{|c|}{ Média depois da EC 95/2016 } \\
\hline & $\mathrm{N}^{\circ}$ de municípios & Proporção (\%) & $\mathrm{N}^{\circ}$ de Municípios & Proporção (\%) \\
\hline $\mathrm{EE}<21,00$ & 0 & - & 0 & - \\
\hline $21,00 \leq \mathrm{EE}<41,00$ & 0 & - & 0 & - \\
\hline $41,00 \leq \mathrm{EE}<60,00$ & 0 & - & 0 & - \\
\hline $61,00 \leq \mathrm{EE}<81,00$ & 7 & 19,44 & 2 & 5,56 \\
\hline $82,00 \leq \mathrm{EE} \leq 100,00$ & 29 & 80,56 & 34 & 94,44 \\
\hline Total & 36 & 100,00 & 36 & 100,00 \\
\hline
\end{tabular}

Como se pode observar, dos 36 municípios analisados, 29 , ou seja, $80,56 \%$, assinalaram os mais elevados escores de eficiência antes da EC, enquanto 34 apresentaram essa performance depois da EC, perfazendo 94,44\%. Esses resultados divergem daqueles encontrados por Nunes e Souza (2019), pois dos 162 municípios cearenses analisados pelos autores, somente sete obtiveram máxima eficiência sob a análise de retornos constantes à escala, perfazendo 4,32\% do total. Sousa et al. (2020) constataram que a eficiência dos sistemas de saúde dos municípios pernambucanos apresentou baixo indicador nas macrorregiões, com média de apenas 0,37, ou seja, os municípios alcançaram $37 \%$ da eficiência dos serviços de saúde possível.

No geral, com base na Tabela 3, constatou-se que houve acrescimento no número de municípios que atingiram os níveis máximo de eficiência média entre os períodos antes e depois da vigência da EC 95/2016.

$\mathrm{Na}$ Tabela 4, observa-se que antes da EC, sete municípios obtiveram os mais altos escores de eficiência; e depois da EC, foram 14 os municípios que obtiveram os mais elevados escores de eficiência média.

Tabela 4: Índice de eficiência dos 36 municípios cearenses mais populosos antes e depois da EC 95/2016

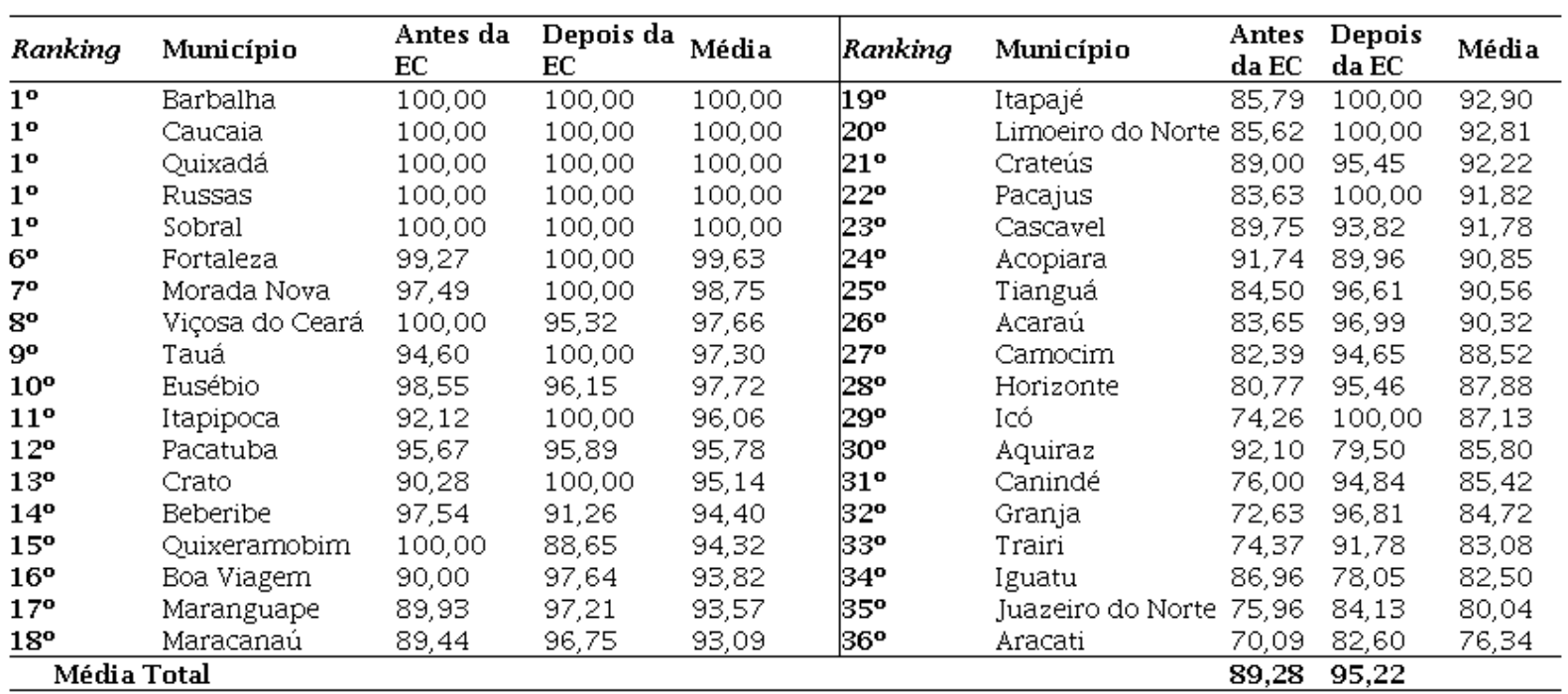


O período depois da EC 95/2016 apresenta maiores escores médios (Tabela 4). Nesse sentido, no geral, o aumento na eficiência dos municípios refuta as predições de Viera e Benevides (2016), que apontaram que a EC 95/2016 criará obstáculos à concretização do direito à saúde, em virtude da escassez da oferta e da baixa produtividade dos serviços públicos.

A maior eficiência não necessariamente ocorre nos municípios que mais gastam com saúde, pois os municípios de Barbalha, Caucaia, Quixadá, Russas, por exemplo, não estão entre os que despenderam mais recursos, entretanto obtiveram os melhores escores.

Queiroz et al. (2013b) fizeram semelhante constatação, já que o município mais ineficiente nos gastos com saúde foi o que realizou o maior dispêndio, sinalizando que a ineficiência do gasto público em saúde está correlacionada com a boa gestão dos recursos.

Três municípios menos eficientes no período antes da EC (Aracati, Granja e Trairi) e três depois (Iguatu, Aquiraz e Aracati), possuem menos de 100 mil habitantes. Em consonância, Andrade et al. (2017) constataram que a capital do Distrito Federal, apesar de ser a terceira mais populosa, assinalou o menor escore de eficiência. Nunes e Souza (2019) também indicaram que o grupo dos municípios cearenses com população acima de 100 mil habitantes registrou o menor índice médio de eficiência técnica do IFDM-Saúde.

\subsection{Nível de produtividade antes e depois da EC 95/2016}

Uma vez analisada a eficiência, verificou-se o comportamento da produtividade total dos municípios ao longo dos períodos, tendo o ano de 2015 como referência (Tabela 5). A produtividade foi avaliada por meio do IPM a partir da mudança de uma DMU entre dois períodos, mediante a análise estatística comparativa (Sánchez, 2018).

Tabela 5: Painel do IPM para os municípios no período de 2016 a 2018

\begin{tabular}{l|c|c|c}
\hline Ano & Variação na eficiência técnica & Variação tecnológica & Produtividade total \\
\hline 2016 & 1,004 & 0,720 & 0,731 \\
2017 & 1,081 & 1,087 & 1,178 \\
2018 & 1,008 & 1,001 & 1,010 \\
\hline
\end{tabular}

Da análise, constatou-se que em 2016 ocorreu diminuição na variação da eficiência técnica, indicando que os municípios aumentaram a eficiência técnica após a EC. Esses achados assemelham-se aos de Duarte e Diniz (2018), que identificaram crescimento de 0,979 para 1,018 na variação da eficiência técnica dos estados brasileiros.

Em relação à variação tecnológica, o acréscimo mais significativo foi em 2017, seguido de 2018. Em 2016, verificou-se variação tecnológica decrescente. De modo geral, constatou-se tendência de queda em 2018, comparando-se a 2017.

Quanto à produtividade total, notou-se, assim como nos outros efeitos, que o aumento mais significativo ocorreu em 2017. Enquanto 2016 apresentou menor escore, 0,731. Ante o exposto, importa ressaltar que foi possível verificar o impacto na regra de financiamento mínimo da saúde a partir da mudança implementada pela EC 86/2015, que fixou percentual escalonado da Receita Corrente Líquida (RCL), no ano de 2016. Ademais, no ano de 2017 (início da vigência da EC 95/2016), verifica-se o aumento da produtividade, pois a regra fixou o percentual de aplicação de $15 \%$ da RCL em ASPS e, a partir de então, teve como piso o gasto do mencionado ano, reajustado pela inflação.

Os impactos da EC podem ser observados em 2018, diante da redução de 14,3\%, se comparado com 2017. Ressalta-se a ocorrência de implicações negativas da EC na produtividade, corroborando os estudos 
que apontam que os maiores impactos ocorrerão ao longo dos 20 anos de sua vigência (Funcia \& Ocké-Reis, 2018; Vairão \& Alves, 2017).

Foram analisados a quantidade de municípios com aumento na eficiência técnica, na variação tecnológica e na produtividade total (IPM) nos anos de 2016 e 2018 (Tabela 6).

Tabela 6: Quantidade de municípios com aumento na eficiência técnica, variação tecnológica e produtividade total

\begin{tabular}{l|c|c}
\hline \multirow{2}{*}{ Discriminação } & \multicolumn{2}{|c}{ Quantidade de municípios } \\
\cline { 2 - 3 } & $\mathbf{2 0 1 6}$ & $\mathbf{2 0 1 8}$ \\
\hline Variação na eficiência técnica & 20 & 25 \\
\hline Variação tecnológica & 3 & 17 \\
\hline Produtividade total (IPM) & 1 & 20 \\
\hline
\end{tabular}

A quantidade de municípios que obtiveram aumento na produtividade total depois da EC, foi bem representativa, sendo $20 \mathrm{em} 2018$ e apenas $1 \mathrm{em}$ 2016. Constatou-se, ainda, que a variação tecnológica foi a principal responsável pela quantidade de municípios com variação positiva depois da EC, pois, em 2016 foram 3 e, em 2018, totalizou 17 municípios. Lembra-se, aqui, que no ano de 2016 a regra de financiamento mínimo da saúde era àquela estabelecida pela EC 86/2015, que fixou percentual escalonado da RCL de 13,2\%. Com a EC 95/2016, para o cálculo do mínimo em ASPS no ano de 2017, foi fixado o percentual mínimo de 15\% da RCL, interferindo no resultado de 2018. Assim, acredita-se que, em linhas gerais, a variação tecnológica foi alterada pela mudança na legislação, consequentemente no financiamento da saúde. Esses achados assemelham-se aos de Ferreira e Silva (2015), pois a evolução produtiva foi afetada em maior parte pela variação tecnológica, tendo em vista que 93,6\% dos municípios potiguares obtiveram aumento nessa variável.

\subsection{Correlação entre a eficiência e os indicadores PIB per capita e IEGM_Saúde}

A discussão dos resultados inicia-se com a estatística descritiva das variáveis de interesse da pesquisa, a eficiência dos gastos públicos em saúde e os indicadores PIB per capita e IEGM_Saúde, referentes ao exercício de 2018 (Tabela 7).

Tabela 7: Estatística descritiva das variáveis de interesse

\begin{tabular}{lcccc}
\hline Variável & Mínimo & Máximo & Média & Desvio-padrão \\
\hline Eficiência & 79,00 & 100,00 & 95,31 & 6,989 \\
PIB per capita & $6.692,00$ & 46830,00 & 15370,36 & 9193,807 \\
IEGM_Saúde & 51 & 92 & 73,72 & 9,407 \\
\hline
\end{tabular}

Antes de examinar a correlação entre as variáveis, faz-se necessário verificar a normalidade dos dados. Para tanto, utilizou-se o teste de Kolmogorov-Smirnov, cuja hipótese nula é que as distribuições se assemelham à normal. Os resultados apontaram que as variáveis eficiência e PIB per capita não apresentam distribuição normal ( -value < 0,05), enquanto a variável IEGM_Saúde apresenta distribuição normal.

A Tabela 8 apresenta os resultados do teste de correlação entre as variáveis eficiência dos gastos públicos em saúde e os indicadores IEGM_Saúde e PIB per capita. Optou-se por utilizar correlação de Pearson com correção bootstrap, a fim de melhorar a inferência estatística das medidas de eficiência. Para interpretar a correlação, utilizaram-se as referências propostas por Levin e Fox (2004), adotadas por Queiroz et al. (2013a). 
Tabela 8: Análise de correlação

\begin{tabular}{|c|c|c|c|c|}
\hline Variável & Item & Eficiência & IEGM_Saúde & PIB per capita \\
\hline Eficiência & $\begin{array}{l}\text { Coeficiente de correlação } \\
\text { p-value }\end{array}$ & 1,000 & & \\
\hline IEGM_saúde & $\begin{array}{l}\text { Coeficiente de correlação } \\
\text { p-value }\end{array}$ & $\begin{array}{l}-, 066 \\
, 703\end{array}$ & 1,000 & \\
\hline PIB per capita & $\begin{array}{l}\text { Coeficiente de correlação } \\
\text { p-value }\end{array}$ & $\begin{array}{l}, 029 \\
, 867\end{array}$ & $\begin{array}{l}, 126 \\
, 465\end{array}$ & 1,000 \\
\hline
\end{tabular}

Destaca-se, inicialmente, que as variáveis PIB per capita e IEGM_Saúde apresentam uma fraca correlação positiva. Esse fato indica que elas vêm captando efeitos distintos. Consequentemente, ambas podem ser utilizadas para aferir a correlação com a eficiência.

Verificou-se que não há correlação entre a eficiência e o PIB per capita, ao nível de $5 \%$ de significância. Aliás, nenhuma evidência pode ser inferida sobre a correlação entre a eficiência na gestão da saúde nos 36 municípios pesquisados e o IEGM_Saúde.

Embora seja mais comum na literatura nacional a indicação de correlação positiva entre os escores de eficiência e o PIB per capita, como em Barbosa e Sousa (2015), os resultados alcançados neste estudo corroboram aqueles encontrados por Asandului et al. (2014) e Nunes e Souza (2019) para a saúde em países europeus e em municípios cearenses, respectivamente. Assim, infere-se que não obrigatoriamente os municípios com situação econômica mais favorável, absorvida pelo PIB per capita, vêm gerenciando mais eficientemente os recursos públicos da saúde, bem como os municípios com melhores IEGM_Saúde também não são mais eficientes, considerando as variáveis do estudo.

\section{CONCLUSÃO}

O estudo analisou a eficiência e a produtividade dos gastos, das ações e dos serviços de saúde nos municípios mais populosos do Ceará, antes e depois da EC 95/2016. Examinou-se, ainda, a correlação entre o nível de eficiência dos municípios e os indicadores de desenvolvimento social PIB per capita e IEGM_Saúde. Para tanto, utilizou-se a DEA e o IPM para calcular a eficiência e a produtividade em saúde nos municípios, respectivamente, nos exercícios de 2015 a 2018. Verificou-se, também, a possível correlação entre o escore de eficiência e os indicadores de desenvolvimento social, no caso o PIB per capita e o IEGM_Saúde referentes a 2018.

Por meio da estimação do modelo DEA, constatou-se, no geral, que o período depois da EC 95/2016 apresentou maior quantidade de municípios que obtiveram melhores índices de eficiência e indicou maiores escores médios. Ademais, constatou-se elevados níveis de eficiência dos municípios, mesclando entre mais e menos populosos.

Antes da EC ocorreu diminuição na variação da eficiência técnica dos municípios em relação ao período depois da EC, indicando que os municípios aumentaram a eficiência técnica após a EC. Em relação à variação tecnológica, o acréscimo mais significativo foi em 2017, seguido do ano de 2018. No ano de 2016, verificou-se variação tecnológica decrescente. No tocante à produtividade total, notou-se, assim como nas outras análises, que o aumento mais significativo ocorreu em 2017, sendo que em 2016 não houve variação positiva na produtividade. Contudo, adverte-se que os resultados por ano indicam que houve uma redução na produtividade média no ano 2018 , corroborando os achados de outras pesquisas que indicam que os maiores impactos do NRF, instituído pela EC 95/2016, ocorrerão ao longo dos 20 anos de vigência da norma (Funcia \& Ocké-Reis, 2018; Rasella et al., 2018).

As variáveis PIB per capita e IEGM_Saúde apresentaram uma fraca correlação positiva. Entretanto, não há correlação entre a eficiência e o PIB per capita e o IEGM_Saúde ao nível de 5\% de significância. Assim, não 
obrigatoriamente os municípios com situação econômica mais favorável, absorvida pelo PIB per capita, vêm gerenciando mais eficientemente os recursos públicos da saúde, bem com os melhores IEGM_Saúde também não são mais eficientes, considerando as variáveis do estudo.

Diante do exposto, entende-se que o estudo pode contribuir para enriquecer a literatura e para subsidiar a administração pública municipal, já que foram feitas análises comparativas entre os dois primeiros anos de aplicação da EC 95 e seus achados podem ajudar na formulação de políticas públicas de saúde no Ceará e em outros estados. Ademais, na análise de correlação foram considerados indicadores de desenvolvimento social com uma abordagem multidimensional, como é o caso do PIB per capita e do IEGM_Saúde.

A pesquisa enfrenta algumas limitações, que devem ser avaliadas no tocante à generalização dos resultados e inferências apresentados. A insuficiência de informações para possibilitar a ampliação do número de outputs e a ausência de indicadores socioeconômicos, foram as principais limitações. Para futuros estudos, sugere-se a investigação da eficiência e da produtividade dos municípios em outras funções. Recomenda--se a utilização de outras variáveis de inputs (como gastos com educação e segurança pública) e outputs (outros indicadores assistenciais, atendimento e sociais) nos modelos DEA e IPM, com o objetivo de ampliar as dimensões estudadas. Além disso, sugere-se que estudos futuros possam investigar as dimensões aqui apresentadas, ampliando-se a série temporal para verificar os impactos da EC 95.

\section{REFERENNCIAS}

Alesina, A., Bariero, O., Fávero, G., Giavazzi, F., \& Paradisi, D. (2017). The effects of fiscal consolidations: Theory and evidence. National Bureau of Economic Research.

Alves, L. A., \& Costa, L. D. (2013). Avaliação da eficiência na atenção básica à saúde nos municípios do estado do Espírito Santo. Anais do Encontro da Associação Nacional de Pós-Graduação e Pesquisa em Administração, Rio de Janeiro, RJ, Brasil, 37.

Andrade, B. H. S., Serrano, A. L. M., Bastos, R. F. S., \& Franco, V. R. (2017). Eficiência do gasto público no âmbito da saúde: uma análise do desempenho das capitais brasileiras. Revista Paranaense de Desenvolvimento, 38(132), 163-179.

Andrett, M. C. S., Lunkes, R. J., Rosa, F. S., \& Brizolla, M. M. B. (2018). Eficiência dos gastos públicos em saúde no Brasil: estudo sobre o desempenho de estados brasileiros. Revista de Gestão em Sistemas de Saúde, 7(2), 114-128.

Araújo, L. C., Bezerra Filho, J. E., Teixeira, A., \& Motoki, F. (2021). Gestões municipais no Brasil: Um estudo a partir do Índice de Efetividade das Gestôes Municipais - IEGM. Revista Catarinense da Ciência Contábil, 20(1), 1-17.

Asandului, L., Roman, M., \& Fatulescu, P. (2014). The efficiency of healthcare systems in Europe: a data envelopment analysis approach. Procedia Economics and Finance, 10, 261-268.

Banker, R. D., Charnes, H., \& Cooper, W. W. (1984). Some models for estimating technical and scale inefficiencies in data envelopment analysis. Management Science, 30(9), 1078-1092.

Barbosa, W. F., \& Sousa, E. P. (2015). Eficiência técnica e de escala do sistema único de saúde nos municípios do nordeste brasileiro. Revista Econômica do Nordeste, 46(3), 99-113.

Beuren, I. M., Moura, G. D., \& Kloeppel, N. R. (2013). Práticas de governança eletrônica e eficiência na utilização das receitas: uma análise nos estados brasileiros. Revista de Administração Pública, 47(2), 421-441.

Bogetoft, P., \& Otto, L. (2011). Benchmarking with DEA, SFA, and R (1st ed., Vol. 157). New York: Springer New York.

Carvalho, G. (2013). A saúde pública no Brasil. Estudos Avançados, 27(78), 7-26.

Charnes, A., Cooper, W. W., \& Rhodes, E. (1978). Measuring the efficiency of decision making units. European Journal of Operational Research, 2(6), 429-444.

Constituição da República Federativa do Brasil. (1988). Brasília: Senado.

Costa, G. K. O., \& Rodrigues, F. D. (2016). Aplicação da análise envoltória de dados para avaliar a eficiência das unidades de saúde da família no município de Natal/RN. Métodos e Pesquisa em Administração, 1(2), 34-44. 
Costa, N. R., \& Lago, J. (2020). A disponibilidade de leitos em unidade de tratamento intensivo no SUS e nos planos de saúde diante da epidemia da COVID-19 no Brasil. Nota Técnica. Fundação Oswaldo Cruz (FIOCRUZ).

Debreu, G. (1951). The coefficient of resource utilisation. Econometrica, 19(3), 273-292.

Duarte, J. M. S., \& Diniz, J. A. (2018). Gastos públicos e produtividade nos serviços de saúde de média e alta complexidade nos estados brasileiros. Revista de Educação e Pesquisa em Contabilidade, 12(4), 450-467.

Emenda Constitucional n. 29, de 13 de setembro de 2000. (2000). Altera os arts. 34, 35, 156, 160, 167 e 198 da Constituição Federal e acrescenta artigo ao Ato das Disposições Constitucionais Transitórias, para assegurar os recursos mínimos para o financiamento das ações e serviços públicos de saúde. Recuperado em 12 dezembro, 2019, de http://www.planalto.gov.br/ccivil_03/constituicao/Emendas/Emc/emc29.htm

Emenda Constitucional n. 95, de 15 de dezembro de 2016. (2016). Altera o Ato das Disposições Constitucionais Transitórias, para instituir o Novo Regime Fiscal, e dá outras providências. Brasília, DF. Recuperado em 12 dezembro, 2019, de http://www.planalto.gov.br/ccivil_03/constituicao/Emendas/Emc/emc95.htm

Farrel, M. J. (1957). The measurement of productive efficiency. Journal of the Royal Statistical Society: Series A (General), 120(3), 253-290.

Flach, L., Mattos, L. K, \& Mendes, V. G. (2017) Eficiência dos gastos públicos em saúde nos municípios do Espírito Santo: um estudo com Análise Envoltória de Dados e Regressão Tobit. Anais do Congresso Brasileiro de Custos, Florianópolis, PR, Brasil, 24.

Ferreira, F. D. S., \& Silva, J. L. M. (2015). Eficiência e produtividade dos gastos públicos no ensino fundamental. Uma análise de convergência dos municípios potiguares. Revista Economia e Desenvolvimento, 14(2), 196-214.

Fonseca, P. C., \& Ferreira, M. A. M. (2009). Investigação dos níveis de eficiência na utilização de recursos no setor de saúde: uma análise das microrregiões de Minas Gerais. Saúde e Sociedade, 18(2), 199-213.

Funcia, F., \& Ocké-Reis, C. O. (2018). Efeitos da política de austeridade fiscal sobre o gasto público federal em saúde. In Rossi, P.; Dweck, E.; Oliveira, A. L. M. (Org.). Economia para poucos: impactos sociais da austeridade e alternativas para o Brasil. (pp. 83-97). São Paulo: Autonomia Literária.

Galoni, D., \& Camargo, L. S. G. (2016). Análise da eficiência econômica dos dez maiores e dez menores municípios paulistas, em termos de arrecadação de tributos, valendo-se da análise por envoltória de dados (DEA) no período de 2012 e 2013. Revista de Finanças e Contabilidade da Unimep, 3(1), 1-18.

Instituto Brasileiro de Geografia e Estatística. (2019). Resolução n. 3, de 26 de agosto de 2019. Recuperado em 30 abril, 2021, de https://www.in.gov.br/en/web/dou/-/resolucao-n-3-de-26-de-agosto-de-2019-212912380

Kohl, S., Schoenfelder, J., Fügener, A., \& Brunner, J. O. (2019). The use of data envelopment analysis (DEA) in healthcare with a focus on hospitals. Health Care Management Science, 22(2), 245-286.

Lei Complementar n. 141, de 13 de janeiro de 2012. Brasília, DF, Senado Federal.

Leite, G. A., Filho, \& Fialho, T. M. M. (2015). Relação entre indicadores de qualidade da gestão pública e de desenvolvimento dos municípios brasileiros. Cadernos Gestäo Pública e Cidadania , 20(67), 277-295.

Levin, J., \& Fox, J. A. (2004). Estatística para ciências humanas (9a ed.). São Paulo: Prentice Hall.

Lobo, M. S. C., \& Araújo, E. C. (2017). Efficiency analysis of public health spending in Brazilian capitals using network data envelopment analysis. Central European Review of Economics and Management, 1(4), 147-173.

Lopes, L. S., Toyoshima, S. H., \& Gomes, A. P. (2009). Determinantes sociais da saúde em Minas Gerais: uma abordagem empírica. Anais do Encontro Nacional da Associação Brasileira de Estudos Regionais e Urbanos, São Paulo, SP, Brasil, 7.

Macêdo, F. F. R. R., Reis, M. R. G., Silveira, M. A., \& Salla, N. M. G. (2019). Transparência fiscal e eficiência dos gastos públicos em educação e saúde: estudo nos municípios cearenses. Anais do Congresso Brasileiro de Custos, Curitiba, PR, Brasil, 26.

Machado, S. P., Jr, Irffi, G., \& Benegas, M. (2011). Análise da eficiência técnica dos gastos com educação, saúde e assistência social dos municípios cearenses. Planejamento e Políticas Públicas, 36, 87-113.

Marinho, A. (2003). Avaliação da eficiência técnica nos serviços de saúde nos municípios do Estado do Rio de Janeiro. Revista Brasileira de Economia, 57(2), 515-534. 
Marinho, E., \& Ataliba, F. (2000). Avaliação do crescimento da produtividade e progresso tecnológico dos estados do Nordeste com o uso da fronteira de produção estocástica. Pesquisa e Planejamento Econômico, 30(3), 427-452.

Mattos, E., Rocha, F., Novaes, L., Arvate, P., \& Orellano, V. (2009). Economia de escala na oferta de serviços públicos de saúde: um estudo para os municípios paulistas. Revista Economia, 10(2), 357-386.

Mazon, L. M., Mascarenhas, L. P. G., \& Dallabrida, V. R. (2015). Eficiência dos gastos públicos em saúde: desafio para municípios de Santa Catarina, Brasil. Saúde e Sociedade, 24(1), 23-33.

Militão, E., \& Mazieiro, G. (2020). Governo Bolsonaro reduz gastos com campanhas de incentivo a vacinação. Portal Uol, Brasília, Recuperado em 12 de janeiro, 2021, de https://noticias.uol.com.br/saude/ultimas-noticias/redac ao/2020/12/26/governo-bolsonaro-reduz-gastos-campanhas-vacinacao.amp.htm?_twitter_impression=true.

Mont Mor, D. S., \& Barbosa, F. S. (2018). Consórcios públicos de saúde: as aderências pelos municípios e o atingimento da eficiência dos serviços prestados. Revista Gestão \& Saúde, 9(3), 379-392.

Nunes, E. S., \& Sousa, E. P. (2019). Eficiência no gerenciamento público com a saúde para os municípios cearenses. Revista Brasileira de Estudos Regionais e Urbanos, 13(1), 98-118.

Peña, C. R. (2008). Um modelo de avaliação da eficiência da administração pública através do método análise envoltória de dados (DEA). Revista de Administração Contemporânea, 12(1), 83-106.

Queiroz, D. B., Nobre, F. C., Silva, W. V., \& Araújo, A. O. (2013a). Transparência dos municípios do Rio Grande do Norte: avaliação da relação entre o nível de disclosure, tamanho e características socioeconômicas. Revista Evidenciação Contábil \& Finanças, 1(2), 38-51.

Queiroz, M. F. M., Silva, J. L. M., Figueiredo, J. S., \& Vale, F. F. R. (2013b). Eficiência no gasto público com saúde: uma análise nos municípios do Rio Grande do Norte. Revista Econômica do Nordeste, 44(3), 761-776.

Ramos, M. O., Silva, M. V. D. C., Cerqueira, A. J. F., \& Queiroz, K. F. F. (2021). A participação social importa na qualidade da gestão pública? Um estudo sobre a efetividade da gestão pública dos municípios da região metropolitana de Salvador-Bahia. Cadernos de Finanças Públicas, 21(1), 1-24.

Rasella, D., Basu, S., Hone, T., Paes-Sousa, R., Ocké-Reis, C. O., \& Millett, C. (2018). Child morbidity and mortality associated with alternative policy responses to the economic crisis in Brazil: A nationwide microsimulation study. PLOS Medicine. Maio.

Rosano-Peña, C., Albuquerque, P. H. M., \& Márcio, C. J. (2012). A eficiência dos gastos públicos em educação: evidências georreferenciadas nos municípios goianos. Economia Aplicada, 16(3), 421-443.

Rosano-Peña, C., Albuquerque, P. H. M., \& Daher, C. E. (2012). Dinâmica da produtividade e eficiência dos gastos na educação dos municípios goianos. Revista De Administração Contemporânea, 16(6), 845-865.

Sánchez, J. J. V. (2018). Malmquist Index with time series to data envelopment analysis. In: Salomon, V. A. P. (Ed.). Multi-criteria methods and techniques applied to supply chain management. Croatia: IntechOpen.

Santos, R. R., Freitas, M. M., \& Flach, L. (2015). Análise envoltória de dados como ferramenta de avaliação da eficiência dos gastos públicos com educação dos municípios de Santa Catarina. Anais do Congresso Brasileiro de Custos, Foz do Iguaçu, PR, Brasil, 22.

Scarpin, J. E., Macedo, F. F. R. R., Starosky, L., Filho, \& Rodrigues, M. M., Júnior. (2012). Análise da eficiência dos recursos públicos direcionados à educação: estudo nos municípios do Estado de Santa Catarina. Revista Gestão Pública: Práticas e Desafios, 3(2), 27-48.

Silva, C. R. M., Alves, R. M. P., Luca, M. M. M., \& Vasconcelos, A. C. (2019). Eficiência da alocação de recursos públicos nas unidades da federação nos governos Lula e Dilma. Cadernos Gestão Pública e Cidadania, 24(78), 1-20.

Silva, F. L. G. (2010). Análise da eficiência técnica dos gastos públicos com saúde no Estado do Ceará. Dissertação de mestrado, Universidade Federal do Ceará, Fortaleza, CE, Brasil.

Silva, M. Z., Moretti, B. R., \& Schuster, H. A. (2016). Avaliação da eficiência hospitalar por meio da análise envoltória de dados. Revista de Gestão em Sistemas de Saúde , .(2), 100-114.

Sousa, K. M., Pinhanez, M. M. S. F., Monte, P. A., \& Cavalcante, P. R. N. (2020). Financiamento, composição dos gastos e eficiência na saúde dos municípios pernambucanos. Administração Pública e Gestão Social, 12(3), 1-20. 
Antonio Rafael Valério de Oliveira, et al. Eficiência e Produtividade dos Gastos, Ações e Serviços...

Sun, D., Ahn, H., Lievens, T., \& Zeng, W. (2017). Evaluation of the performance of national health systems in 2004-2011: an analysis of 173 countries. Plos One, 12(3), 1-13.

Teles, J. S. (2018). Eficiência relativa da gestão de saúde nos municípios do Estado do Ceará. Dissertação de mestrado, Universidade Federal do Ceará, Fortaleza, CE, Brasil.

Tribunal de Contas do Estado do Ceará. (2019). Índice de efetividade da gestão municipal (IEGM) - 2018, apuração 2019. Recuperado em 05 maio, 2021, de https://www.tce.ce.gov.br/cidadao/consulta-de-processos?nrProc=00 455/2019-0

Vairão, N. S. Jr., \& Alves, F. J. D. S. (2017). A Emenda Constitucional 95 e seus efeitos. Revista de Contabilidade do Mestrado em Ciências Contábeis da UERJ, 22(2), 54-75

Vieira, F. S., \& Benevides, R. P. S. (2016). O direito à saúde no Brasil em tempos de crise econômica, ajuste fiscal e reforma implícita do Estado. Revista de Estudos e Pesquisas sobre as Américas, 10(3), 1-28.

Zhang, Y., Wang, Q., Jiang, T., \& Wang, J. (2018). Equity and efficiency of primary health care resource allocation in mainland China. Int J Equity Health, 17(140), 1-12. 